\title{
Robots, Aesthetics, and the Heritage Context
}

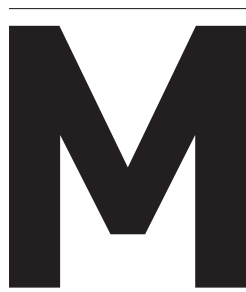

ost people have not yet interacted directly with a robot in their everyday lives except maybe with children's toys or those charming robotic vacuum cleaners. While there are ongoing experiments with robots in healthcare, many more are employed in hightech, efficient environments such as factories. But robots have also played a large part in our cultural and historical imagination. This is celebrated in recent exhibitions such as "Robots" at the Science Museum in London and "Hello, Robot. Design Between Human and Machine" at the Vitra Design Museum in Weil am Rhein, Germany. Apart from such exhibits and despite robots' pointing toward future automation, robots are starting to be used in heritage contexts to provide services, from remote exploration of museum sites to robot-based guided tours. But what happens when robots move from being an attraction to being an agent that shares physical space with people?

Beyond the laboratory, everyday contexts require robots to be incredibly stable and reliable over time. This is particularly important for historical sites. The use of robots should not, under any circumstances, damage the heritage or endanger the safety of staff or visitors, or interfere with preservation or visitor enjoyment and appreciation. However, challenges emerging from the use of robots in heritage contexts relate not only to functional aspects but also perceptual ones.

\section{THE ROLE OF AESTHETICS}

Among the many factors that can determine a robot's acceptability, such as purpose or safety, its physical appearance can instantly affect how users perceive it. Therefore, it is of primary importance that the robot be designed in such a way that its skills and functions are quickly and easily communicated, since that determines whether people will understand its role and respond appropriately.

However, effectively communicating its function doesn't ensure a robot's success. For instance, in the case of service environments, robots share physical contexts with people through activities and interactions, social relationships, and artifacts. Through all these aspects, a robot automatically establishes implicit relationships that offer cues to people as they form interpretations and judgments. Cleaning robots, for instance, introduce novel technologies for cleaning activities that people are already doing with vacuum cleaners. Beyond being employed for a clear function, a robot's appearance and aesthetics remind people of existing products. This approach attempts to avoid ambiguities and concerns. However, the employment of humanoid robots for jobs that are usually entrusted to people, such as museum guides, customer care, or home assistance, will automatically bring forth a comparison between robot and

The design of a robot should take into account the particularities of the specific context in which it will be located. human. This comparison can surface uncanny feelings and concerns. Furthermore, even when people are not replaced, robots are evaluated on their ability to fit into people's sociocultural context. The design of a robot, then, should take into account the particularities of the specific context in which it will be located.

Given these considerations, a good practice for the design of a robot is a formal synthesis that combines a morphology with an explicit function and the aesthetics familiar to the context.

\section{VIRGIL}

In 2015, I was part of a team at Politecnico di Torino that designed Virgil, a telepresence robot, in response to these considerations of morphology and familiar aesthetics. The design, consisting of a robot that allows the remote exploration of inaccessible areas, was used at the Castle of Racconigi in the Piedmont region of Italy. It resulted from a broad reflection on the dual nature of cultural heritage, as a specific context, to focus not only on preservation but also on enjoyment and accessibility for visitors. The castle, one of the royal residencies of the Savoy family, is a context rich in artwork, artifacts from daily life, and architecture that preserves a nearly thousand-year history. Guided by Professor Claudio Germak, our team designed an application within the context of a project sponsored by Telecom Italia Mobile in collaboration with the Terre dei Savoia association.

Our aim was to provide visitors with remote access to inaccessible areas of the heritage, such as the nurses' rooms, the tiepidarium 


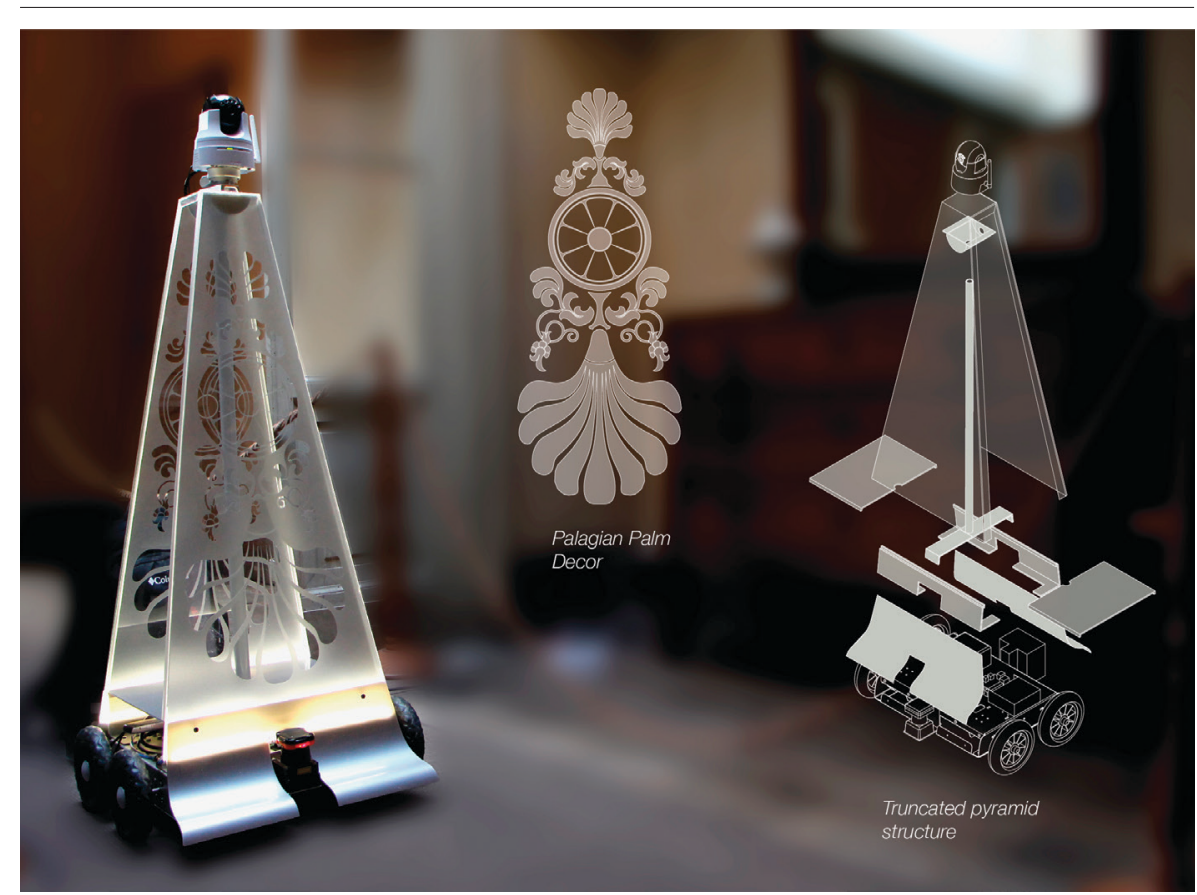

The robot Virgil inside an area of the Castle of Racconigi. The illustrations show the two main characteristics of the robot's appearance: the shape and decoration.

(thermal bath), and other areas currently excluded from the exhibit due to issues of safety, restoration, or cataloging. To do so, a museum guide used a Web application to control a robot. Apart from the willingness to provide an extension and enhancement of the heritage visit, we devoted great attention to the possible perceptual drawbacks. We focused on how to communicate the particular role and function of the robot and how to define an aesthetics appropriate for that specific context. Regarding the first point, we chose to keep the main elements, such as wheels, laser scanner, and camera, visible so that the function of the robot was explicit. Regarding the familiar aesthetics, we analyzed the heritage context from a physical and cultural point of view. This led to a robot design that, in both form and decoration, recalls existing elements of the heritage.

The cover, made of PMMA (poly-methyl-methacrylate), has the form of a truncated pyramid. This morphology was chosen to recall similar shapes, widely found in Savoy tradition, used in obelisks, bollards, and other architectural elements or furniture. The choice of a transparent material was determined by the need to ensure maximum lightness from both a structural and an aesthetic point of view.

Furthermore, the body of the robot was decorated with the aim of customizing it in relation to the context. It consists of decor, which represents the Palagiana palm, an existing decoration that can be found in many artifacts of the castle. The decoration, then, was both a way to ensure aesthetic coherence and a tribute to both the architect and the history of a place characterized by continuous evolution over centuries.

This design approach represented a way to familiarize visitors with the concept of remote access to otherwise inaccessible areas. It also revealed greater design opportunities for considering the aesthetics of robotics that went beyond their functionality and acceptability.

\footnotetext{
(1) Maria Luce Lupetti is a Ph.D. candidate in design for service robotics at Politecnico di Torino, Italy. Her research, focused on humanrobot interaction and play, is supported by Telecom Italia Mobile (TIM). During her Ph.D. she has been publicity chair for HRI Pioneers Workshop 2017 and a visiting scholar at $X$-Studio in the Academy of Art and Design at Tsinghua University, China.

$\rightarrow$ d033947apolito.it
}

\section{Call for Nominations} for ACM General Election

The ACM Nominating Committee is preparing to nominate candidates for the officers of ACM:

President, Vice-President, Secretary/Treasurer; and two

Members at Large.

Suggestions for candidates are solicited. Names should be sent by November 5, 2017 to the Nominating Committee Chair, c/o Pat Ryan,

Chief Operating Officer,

ACM, 2 Penn Plaza, Suite 701,

New York, NY 10121-0701, USA.

With each recommendation, please include background information and names of individuals the Nominating Committee can contact for additional information if necessary.

Alexander L. Wolf is the Chair of the Nominating Committee, and the members are

Karin Breitman, Judith Gal-Ezer, Rashmi Mohan, and Satoshi Matsuoka.

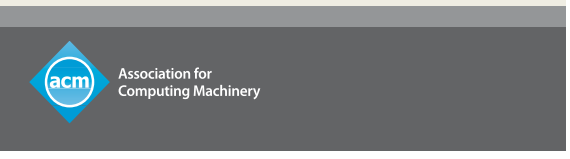

